\title{
New Japanese classifications and treatment guidelines for gastric cancer: revision concepts and major revised points
}

\author{
Takeshi Sano • Takashi Aiko
}

Published online: 15 May 2011

(C) The International Gastric Cancer Association and The Japanese Gastric Cancer Association 2011

\section{Introduction}

In 2010, the Japanese Gastric Cancer Association (JGCA) published new versions of both the Japanese Classification of Gastric Carcinoma (JC) and the Japanese Gastric Cancer Treatment Guidelines (JGL). This was the first integrated revision of the two systems, implementing major structural changes in each. The primary aim of the revision is to provide clinicians and researchers worldwide with a comprehensive and updated guide to the diagnosis and treatment of gastric cancer. English editions of the two systems are now available in this journal $[1,2]$.

\section{History of the JC and JGL}

The first edition of the JC (named "General Rules for Gastric Cancer Study") was published in 1962 to standardize the surgical and pathological documentation of gastric cancer. At that time, the International Union Against Cancer (UICC) and the American Joint Committee on Cancer (AJCC) had not yet established a staging system for gastric cancer. Since then, the JGCA (formerly the Japanese Research Society of Gastric Carcinoma) has

For the Japanese Gastric Cancer Association.

The online version of the two main articles referred to this prefatory article can be found under doi:10.1007/s10120-011-0041-5, and doi:10.1007/s10120-011-0042-4.

T. Sano $(\square)$

The Cancer Institute Hospital, Tokyo, Japan

e-mail: takeshi.sano@jfcr.or.jp

T. Aiko

Kagoshima University, Kagoshima, Japan made periodic revisions and expanded the JC into an original comprehensive guide covering all aspects of the diagnostic and therapeutic procedures for the disease, ranging from the handling of resected specimens for pathological investigation to the extent of lymphadenectomy. It has become customary in Japan to record all cases of gastric cancer in hospital databases in accordance with the JC. Three English editions of the JC were published, corresponding to the 10th, 12th, and 13th Japanese editions, in 1981, 1995, and 1998, respectively [3-5].

In 2001, the JGCA launched the first edition of the treatment guidelines apart from the JC [6]. The primary aim of the JGL was to provide general as well as specialized clinicians with knowledge on standard treatments, based on evidence where available, and consensus, so that a patient with gastric cancer could be offered such treatments anywhere in the country. Because novel treatment modalities and novel handling of clinical issues have constantly been proposed in Japan, the JGL proposed two independent lists of stage-specific treatments; a standard list and an investigational list. This concept has been gradually and widely accepted in the clinical scene and has changed the general practice in Japan. A patients' version of the JGL was also published and has been used to enhance understanding of the treatment of gastric cancer in the general public. The second edition of the JGL was published in 2004, with minor modifications.

\section{Concept of the integrated revision of the JC and JGL in 2010}

The time for revision of the JC and JGL coincided for the first time in 2010. On this occasion, the JGCA committees agreed with the following concepts: 
(a) The domains to be dealt with by the JC and the JGL should be clearly separated as follows:

- The JC provides the basic rules to describe the status of a tumor (primary and metastatic tumor, stage grouping, histology, etc.) and the evaluation of treatment outcome (response evaluation, amount of residual disease, etc.), and will not be revised for 10 years.

- The JGL defines the treatment methods (extent of lymphadenectomy, endoscopic resection, etc.), clarifies the indications for and details of each treatment (algorithm, chemotherapy regimens, etc.), and will be revised every 2-3 years. New important evidence requiring modification of the standard therapy will be announced on the website of the JGCA.

(b) For the description of tumor status in the JC (T/N/M categories, stage grouping, etc.), definitions identical with those in the UICC/TNM 7th edition [7] are adopted so that the Japanese experience can be expressed using the international terminology.

\section{Major points revised in the JC}

\subsection{From the Japanese traditional T/N/M to the international $\mathrm{T} / \mathrm{N} / \mathrm{M}$}

The significations of the T/N/M categories in the new JC are identical to those in the UICC/TNM 7th edition. The modification of the $\mathrm{N}$ category is the largest change not only in this revision but also in the whole history of the JC. Traditionally the lymph node stations in the gastric drainage area were classified into three groups (or four in some editions) depending on the anatomical position of the station in relation to the location of the primary tumor, and these numbers were also used to express the grade of nodal metastasis (N1-3) and the extent of lymphadenectomy (D1-3); e.g., cancer with metastasis to a second group node was designated as N2, and complete dissection of up to the second group nodes was defined as D2. This rule was consistent throughout the history of the $\mathrm{JC}$, though details regarding classification of the nodal groups had been modified in each edition. In the new version of the JC, this nodal grouping has been abandoned, and the $\mathrm{N}$-number solely signifies the grade of nodal metastasis in terms of the number of metastatic lymph nodes as determined in the UICC/TNM 7th edition. Naturally, the extent of lymphadenectomy had to be newly defined independently from the N-category in the new JGL.

Until the current revision of the JC, the JGCA designated hepatic and peritoneal metastases as $\mathrm{H} 1$ and $\mathrm{P} 1$ and treated them separately from other distant metastasis (M1). Thus, some cases had been recorded as T3N2MOH1P1. In the new version, the M-category is equivalent to that of the UICC/TNM 7th edition and includes all distant metastasis. The designations of " $\mathrm{H}$ " and " $\mathrm{P}$ ", however, are clinically useful and thus remain in the new JC as a subclass of the M-category.

\subsection{Stage}

The same stage grouping as that in the UICC/TNM 7th edition has been adopted in the new JC. It should be noted that survival analyses of a large number of the Japanese and Korean patients contributed to the determination of this UICC/TNM stage grouping [8].

\subsection{Definition of the esophagogastric junction (EGJ) area}

Although the JC basically complied with the staging system proposed in the UICC/TNM 7th edition, the JGCA Committee firmly denied the new UICC/TNM definition of EGJ tumors. We remain more comfortable to consider adenocarcinomas of the subcardia (Siewert type 3) as gastric cancer and believe that these should be classified and staged using the gastric scheme; not the esophageal scheme as in the UICC/TNM 7th edition. In the new JC, we adopted the definition of the EGJ area proposed by the Japan Esophageal Society [9], i.e., the area extending $2 \mathrm{~cm}$ above to $2 \mathrm{~cm}$ below the EGJ.

\subsection{From "four findings" to "two classifications"}

In the 1998 edition of the JC [5], clinical and pathological findings were recorded in 4 separate phases as "clinical (preoperative)", "surgical", "pathological", and "final" findings. In order to comply with the UICC/TNM system, the new JC distinguishes only clinical and pathological classifications.

\subsection{Adoption of international criteria of treatment evaluation}

The traditional classification "Resection (Curability) A/B/ $C$ " has been abandoned and has been replaced by "R0/1/2 (residual disease)". Staging after neoadjuvant treatment is expressed with the prefix "y". These changes are in accordance with the UICC/TNM system. For the response evaluation of chemotherapy, the response evaluation criteria in solid tumors (RECIST) version 1.1 has been adopted. 
4.6 Modification of the histological diagnosis of gastric biopsy ("Group Classification")

The "Group Classification" has been widely used to diagnose the specimens obtained by endoscopic biopsy in Japan. The definitions of Groups 2 and 3 have undergone significant modifications.

\section{Major points revised in the JGL}

\subsection{Definition of and indications for lymphadenectomy}

(D)

The terms "D1/D2/D3" were originally defined in the JC and have been widely used worldwide to describe the extent of lymphadenectomy. Most randomized controlled trials (RCTs) of gastric cancer surgery including the Dutch, Medical Research Council (MRC), and Taipei D1/D2 trials [10-12], were conducted using the JC definitions. However, outside these clinical studies, the terms "D1-3" have not always been used with accuracy in the strict sense. It is generally and mistakenly believed outside Japan that the first group nodes are equal to the perigastric nodes and the second group nodes are those along the celiac artery and its branches, and that the dissections of these are designated as D1 and D2, respectively. However, the original definitions of N1-3 and D1-3 are far more complicated [5]: the location of the primary tumor is determined as one of five categories (various combinations of the three equal portions of the stomach), according to which each lymph node station is given a group number $(1,2$, 3, or $\mathbf{M})$. For example, the left paracardial lymph nodes (station No. 2) are classified as group 1 nodes for a tumor located in the upper third of the stomach, but as group 3 nodes for a middle or middle/lower tumor, and as group $M$ nodes (distant metastasis) for a tumor confined to the lower third of the stomach.

As this complicated definition of the nodal groups was established based on the results of detailed efficacy analysis of each lymph node station [13], surgeons would have the best chance to cure patients if they strictly obeyed the rule of D2. However, the grouping was too complicated to be accurately understood worldwide and, in the first place, the tumor location may not have been as correctly categorized by surgeons/pathologists as the JGCA intended to.

In the new JGL, the definition of lymphadenectomy has been remarkably simplified: the lymph node stations to be dissected in D1, D1+, and D2 are defined for total and distal gastrectomy regardless of the tumor location. D3 is no longer defined, because the rationale to recommend this super-extended surgery was lost by the negative results of our own RCT [14].
Apart from the two major types of gastrectomy (total and distal), pylorus-preserving gastrectomy and proximal gastrectomy are proposed as options for early gastric cancers, for each of which D1 and D1+ (but not D2) are defined. It should be noted that the lymph nodes along the left gastric artery (No. 7), which used to be classified as N2 for tumors in any location, are now included in the D1 for any type of gastrectomy. Lymph node station No. 14v, on the other hand, has been excluded from the D2 even for distal tumors.

The JGCA recommends that non-early, potentially curable gastric cancers should be treated by D2 lymphadenectomy. D1 or D1+ should be considered as an option for $\mathrm{T} 1$ tumors. D1+ can be a substitute for D2 in a poor-risk patient or under circumstances where D2 cannot be safely performed.

The JGCA expects that these simplified definitions of lymphadenectomy will help specialized surgeons worldwide to standardize gastrectomy and to obtain the best surgical results.

\subsection{Chemotherapy for metastatic/recurrent gastric cancer}

Several RCTs of chemotherapy for metastatic/recurrent gastric cancer were recently concluded in Japan, and for the first time in the history of the JGL, the JGCA made a recommendation of a first-line regimen for metastatic/ recurrent gastric cancer; namely, S-1 + cisplatin. S-1 monotherapy was recommended for those in whom the use of cisplatin was not indicated. Although S-1 + cisplatin did not show superiority over 5-fluorouracil (FU) + cisplatin in a global randomized trial conducted outside Japan (FLAGS [15]), it has been regarded as the standard regimen for Japanese patients on the basis of two Japanese RCTs, SPIRITS [16] and JCOG9912 [17]. The JGCA also made a statement that it does not recommend the first-line use of irinotecan + cisplatin and S-1 + irinotecan because these regimens did not show superiority over 5-FU monotherapy and S-1 monotherapy, respectively [17, 18].

\subsection{Adjuvant therapy after curative gastrectomy}

Adjuvant chemotherapy with S-1 for patients with pathological stage II or III gastric cancer (according to the JC 13th edition) following curative D2 gastrectomy showed significantly better survival (overall and relapse-free) than surgery alone in the ACTS-GC trial [19]. The JGCA made a prompt announcement of this positive result on its website when the trial was concluded, and the recommendation has been highlighted in the new JGL. Because the definitions of stages II and III have been changed in the new JC, 
the JGL now shows which stages of the new JC correspond to stages II and III in the previous edition.

Although survival benefits of adjuvant chemoradiation therapy and neoadjuvant chemotherapy have been demonstrated in the United States and Europe, evidence is yet to be established in Japan, and the JGL deals with them as investigational treatments.

\section{Perspective}

With this major revision of the JC, the JGCA now shares the staging system of gastric cancer with the UICC and the AJCC. We expect that the vast Japanese experience regarding this disease can now be recorded and reported in the universal language. At the same time, we expect that our simplified definitions of lymphadenectomy in the revised JGL, together with the documentation system in the JC, will provide surgeons all over the world with a standard for recording their surgery and comparing the results. Although the JGL is based primarily on evidence generated from Japanese trials, the JGCA committee is continually looking for emerging evidence throughout the world to prepare for future revisions.

\section{References}

1. Japanese Gastric Cancer Association. Japanese classification of gastric carcinoma: 3rd English edition. Gastric Cancer 2011. doi: 10.1007/s10120-011-0041-5.

2. Japanese Gastric Cancer Association. Japanese Gastric Cancer Treatment Guidelines 2010 (ver. 3). Gastric Cancer 2011. doi: 10.1007/s10120-011-0042-4.

3. Kajitani T, Japanese Research Society for the Study of Gastric Cancer. The general rules for gastric cancer study in surgery and pathology. Jpn J Surg. 1981;11:127-45.

4. Japanese Research Society for Gastric Cancer. Japanese classification of gastric carcinoma. 1st English edition. Tokyo: Kanehara; 1995.
5. Japanese Gastric Cancer Association. Japanese classification of gastric carcinoma: 2nd English edition. Gastric Cancer. 1998;1: $10-24$.

6. Nakajima T. Gastric cancer treatment guidelines in Japan. Gastric Cancer. 2002;5:1-5.

7. International Union Against Cancer. In: Sobin LH, Gospodarowicz, MK, Wittekind C, editors. TNM classification of malignant tumours. 7th ed. New Jersey: Wiley-Blackwell; 2009.

8. Ahn HS, Lee HJ, Hahn S, et al. Evaluation of the seventh American Joint Committee on Cancer/International Union Against Cancer classification of gastric adenocarcinoma in comparison with the sixth classification. Cancer. 2010;116:5592-8.

9. Japan Esophageal Society. Japanese classification of esophageal cancer, tenth edition: parts II and III. Esophagus 2009;6:71-94.

10. Bonenkamp JJ, Songun I, Hermans J, et al. Randomised comparison of morbidity after D1 and D2 dissection for gastric cancer in 996 Dutch patients. Lancet. 1995;345:745-8.

11. Cuschieri A, Fayers P, Fielding J, et al. Post-operative morbidity and mortality after D1 and D2 resections for gastric cancer: preliminary results of the MRC randomised controlled surgical trial. Lancet. 1996;347:995-9.

12. Wu CW, Hsiung CA, Lo SS, et al. Nodal dissection for patients with gastric cancer: a randomized controlled trial. Lancet Oncol. 2006;7:309-15.

13. Sasako M, McCulloch P, Kinoshita T, Maruyama K. New method to evaluate the therapeutic value of lymph node dissection for gastric cancer. Br J Surg. 1995;82:346-51.

14. Sasako M, Sano T, Yamamoto S, et al. D2 lymphadenectomy alone or with para-aortic nodal dissection for gastric cancer. N Engl J Med. 2008;359:453-62.

15. Ajani JA, Rodriguez W, Bodoky G, et al. Multicenter phase III comparison of cisplatin/S-1 with cisplatin/infusional fluorouracil in advanced gastric or gastroesophageal adenocarcinoma study: The FLAGS trial. J Clin Oncol. 2010;28:1547-53.

16. Koizumi W, Narahara H, Hara T, et al. Randomized phase III study of S-1 alone versus $\mathrm{S}-1+$ cisplatin in the treatment for advanced gastric cancer (The SPIRITS trial): a phase III trial. Lancet Oncol. 2008;9:215-21.

17. Boku N, Yamamoto S, Shirao K, et al. Fluorouracil versus combination of irinotecan plus cisplatin versus $\mathrm{S}-1$ in metastatic gastric cancer: a randomized phase 3 study. Lancet Oncol. 2009;10:1063-9.

18. Narahara H, Iishi H, Imamura $H$, et al. Randomized phase III study comparing the efficacy and safety of irinotecan plus S-1 with S-1 alone as first-line treatment for advanced gastric cancer (study GC0301/TOP-002). Gastric Cancer. 2011;14:72-80.

19. Sakuramoto S, Sasako M, Yamaguchi T, et al. Adjuvant chemotherapy for gastric cancer with S-1, an oral fluoropyrimidine. N Engl J Med. 2007;357:1810-20. 